\title{
Guest editorial: WWWJ special issue of the 20th international conference on Web information systems engineering (WISE 2019)
}

\author{
Reynold Cheng ${ }^{1} \cdot$ Nikos Mamoulis $^{2} \cdot$ Xin Huang $^{3}$ \\ Accepted: 9 October 2020 / \\ Published online: 20 October 2020 \\ CC Springer Science+Business Media, LLC, part of Springer Nature 2020
}

\section{WISE 2019}

The 20th International Conference on Web Information Systems Engineering (WISE 2019) was held in Hong Kong, China, during January 19-21, 2020. It marked the 20th anniversary of the conference, which was first held in Hong Kong in 2000. Building on the success of its predecessors, WISE 2019 continued to provide an international forum for researchers, professionals, and industrial practitioners to share their knowledge in the rapidly growing area of Web technologies, methodologies, and applications. The first WISE event took place in Hong Kong, China (2000). Then the trip continued to Kyoto, Japan (2001); Singapore (2002); Rome, Italy (2003); Brisbane, Australia (2004); New York, USA (2005); Wuhan, China (2006); Nancy, France (2007); Auckland, New Zealand (2008); Poznan, Poland (2009); Hong Kong, China (2010); Sydney, Australia (2011); Paphos, Cyprus (2012); Nanjing, China (2013); Thessaloniki, Greece (2014); Miami, USA (2015); Shanghai, China (2016); Puschino, Russia (2017); Dubai, UAE (2018); In 2019, WISE returned to Hong Kong, China, supported by the Hong Kong Polytechnic University and City University of Hong Kong.

This article belongs to the Topical Collection: Special Issue on Web Information Systems Engineering 2019

Guest Editors: Reynold Cheng, Nikos Mamoulis, and Xin Huang

Reynold Cheng

ckcheng@cs.hku.hk

Nikos Mamoulis

nikos@cs.uoi.gr

Xin Huang

xinhuang@comp.hkbu.edu.hk

1 University of Hong Kong, Hong Kong, China

2 University of Ioannina, Ioannina, Greece

3 Hong Kong Baptist University, Hong Kong, China 
A total of 211 research papers were submitted to the conference for consideration, and most of them were reviewed by three reviewers. Finally, 50 submissions were selected as regular papers (with an acceptance rate of 23.7\%) [1,2]. The research papers cover the areas of blockchain, deep learning, machine learning, recommender systems, data mining, Web-based applications, graph learning, knowledge graphs, graph mining, text mining, and crowdsourcing. In addition to regular papers, The WISE-2019 program also featured two workshops: (1) The 6th international workshop on big data quality and trust (QUAT); and (2) The international workshop on Web information systems in the era of AI. In addition, the conference program included two distinguished keynote presentations by Prof. Wei Zhao (American University of Sharjah, UAE) and Prof. Weiyi Meng (State University of New York at Binghamton, USA) respectively.

\section{The special issue}

The six top ranked papers out of 50 full papers at WISE 2019 have been selected for the special issue of World Wide Web Journal (WWWJ). The authors have been asked to extend their conference papers for journal publication and in accordance with customary practice of adding at least $30 \%$ new material. The revised papers again went through the review and revision processes in accordance with WWWJ guidelines.

The first paper by Xin et al. proposes a Locally-Global model (LoG) for entity disambiguation. The proposed LoG extracts global features locally to reduce incorrect entity assignments in a document with multiple-topics. In particular, they develop a novel distance measure CoSimTC based on parse tree to produce mention neighbors. A keyword extraction method Sent2Word is also designed to detect keywords of each document. Moreover, they extend the Graph Attention Network (GAT) to integrate both local and global features to produce a discriminative representation for each candidate entity.

The second paper by Zhu et al. proposes a new CPL framework to combine two techniques of pointwise prediction and Learning to Rank (L2R), which improves the performance of top- $\mathrm{N}$ recommendations. Based on the CPL framework, they implemented one instantiations of CPLmg, which has two components of factorized sparse linear method and graded average precision factor model to perform pointwise prediction and L2R, respectively. Extensive experiments on four real-world datasets show the good performance of the proposed CPLmg method and CPL framework.

The third paper by Huang et al. studies the problem of structural diversity search, which finds the top-k vertices with the largest structural diversity in a graph. They use the discriminative core and h-index to automatically model social contexts without parameters. They propose an efficient top-k search algorithm with an upper bound pruning strategy, and also a parallel top-k search algorithm to simultaneously compute a batch of vertices using multi-threading on large graphs.

The fourth paper by Niu et al. investigates the problem of Knowledge Graph Completion (KGC), which aims at complementing missing relationships between entities in a Knowledge Graph. They propose an open-world KGC model by adopting a Multiple Interaction Attention (MIA) mechanism to model various kinds of entity interactions. Moreover, additional textual features of head entity descriptions and different scoring functions are used to improve the proposed model. Experiments show the superiority of proposed methods on open-world KGC tasks.

The fifth paper by Yan et al. proposes efficient methods to handle conditional queries and data storage on the consortium blockchain platform of Hyperledger Fabric. To improve the 
performance of conditional queries, they build an AUP index and provide the AUP update solutions during transactions. To speed up data storage, they create a cache for the data in the block header for fast MVCC validation and VSCC validation.

The last paper by $\mathrm{Xu}$ et al. studies the problem of transactions with concurrency conflicts on the Hyperledger Fabric blockchain platform. They present two solutions to optimize the transaction throughput of Hyperledger Fabric. The first LMLS method improves the Write-Write Conflict, which uses lock mechanism in the transaction flow and add indexes to improve the ledger storage. The second cache-based method improves the Read-Write Conflict by accelerating reading data and ensuring data consistency.

Acknowledgments We are very indebted to the reviewers who reviewed the papers very carefully. We would also like to thank all the authors who submitted their papers to the special issue. Special thanks to the journal editors for their great help and support in organizing the issue.

\section{References}

1. Cheng, R., Mamoulis, N., Sun, Y., Huang, X. (eds.): Web Information Systems Engineering - WISE 2019 - 20th International Conference, Hong Kong, China, November 26-30, 2019, Proceedings, vol. 11881 of Lecture Notes in Computer Science. Springer, Berlin (2019)

2. U, L.H., Yang, J., Cai, Y., Karlapalem, K., Liu, A., Huang, X. (eds.): Web Information Systems Engineering - WISE 2019 Workshop, Demo, and Tutorial, Hong Kong and Macau, China, January 19-22, 2020, Revised Selected Papers, vol. 1155 of Communications in Computer and Information Science. Springer, Berlin (2020)

Publisher's note Springer Nature remains neutral with regard to jurisdictional claims in published maps and institutional affiliations. 